\title{
FICHA PARA EL INVENTARIO DE UTILES LITICOS
}

Carlos J. Puch Ramírez

Departamento Arqueología de la U. A. M., 1975

La labor de inventario de los materiales arqueológicos que se acumulan en las dependencias de los Museos, Universidades y otros centros, ha sido enfocada desde diferentes puntos de vista, tendentes ya sea a la simple anotación de la pieza acompañada de unos pocos datos más, que, generalmente, siendo insuficientes para una posterior revisión, exigen la consulta de memorias de excavación u otros documentos, o ya sea a la elaboración de una documentación exhaustiva, y no por ello imprescindible, que requiere una paciente labor descriptiva y analítica de cada objeto particular.

Moviéndonos dentro de las directrices de trabajo del Departamento de Prehistoria y Arqueología de la Universidad Autónoma de Madrid, cuya normativa apunta hacia la uniformación del tratamiento de la información suministrada por los hallazgos arqueológicos, hemos elaborado un ficha, cuyo objetivo no va más allá de la acumulación sistemática y ordenada de datos de índole física, bibliográfica y —en sentido lato- analítica, con el único propósito de inventariar el material lítico trabajado. Insistimos en el hecho de que no pretendemos «clasificar» los objetos a través de un ficha, puesto que la labor de clasificación objetiva (para este tipo de material) no es un cometido a corto plazo, sino que la entendemos como el último eslabón de una cadena de procesos y análisis en los que el elemento subjetivo del «clasificador» ha de intervenir en la mínima proporción... Sin embargo, creemos poder aportar una serie de datos que, convenientemente tratados dentro de un proceso de cálculo (mediante un ordenador electrónico), serán de utilidad para una clasificación matemática posterior de los objetos, según diferentes criterios. Estos datos son los recogidos en la tercera página de la ficha sobre la que trata este trabajo. 
Veamos a continuación el contenido de la ficha, examinándola paso a paso:

\section{Primera página}

Hemos repartido los datos en cuatro grupos:

1. Datos de inventario: o de procedencia. En las casillas Coordenada $X$, Coordenada $Y$ y Profundidad $Z$, indicaremos, con respecto a un origen de coordenadas determinado $(X=0, Y=0, Z=0)$, la posición del hallazgo en dos ejes horizontales, X e Y (fijados por ejemplo en el Este y el Norte, o cualesquiera otros ejes predeterminados), y la profundidad $\mathrm{Z}$ sobre un eje vertical, perpendicular a los anteriores, cuyo punto de cota altimétrica $=0$ es el punto de intersección de los tres ejes, todo ello referido al área del yacimiento de que procede el objeto.

2. Datos de Inventario: dentro de este apartado, interesa particularmente, para la anotación de algunos atributos, tales como la materia prima, el color y el estado de rodadura, ceñirse a algunos códigos establecidos de antemano. Así, para el color, utilizaremos el Code Expolaire, para rocas, que consideramos muy adecuado. Lamentablemente, la materia prima no siempre es fácil de determinar por existir variedades y anomalías, incluso en un mismo tipo de roca, difíciles de apreciar a simple vista.

3. Datos analíticos: rechazamos en este apartado toda interpretación morfológica o analítica, basada en un examen a priori del objeto. Para ello limitamos nuestra labor a un examen doble: a) documental: buscaremos interpretaciones diferentes para el objeto, dadas por diversos autores, anotando la referencia bibliográfica correspondiente que, más tarde, a pie de página, en el apartado 4, ampliaremos (título completo, editorial, fecha, etc.). b) analítico —en s. 1.- agrupando las piezas, según su naturaleza, en nodulares y sobre lasca, ambas excluyentes entre sí. No ocurre lo mismo con los atributos de cada uno de estos grupos, así coom los del otro grupo (Levallois, No-Levallois), los cuales pueden darse simultáneamente en una misma pieza. Hagamos hincapié en el hecho de que en este apartado sólo pretendemos dar una idea general del objeto, sin adentrarnos en un examen tipológico.

Siendo, por tanto, una descripción, no se trata de definir, a través de estas casillas, la pieza estudiada, por lo cual acosejamos que, en caso de duda, no se rellenen las mismas. 
4. Bibliografía: ampliación de las referencias bibliográficas, y mención de otras obras relacionadas con el tema.

Hemos excluido intencionalmente el estudio tecnológico del objeto lítico, a pesar de parecernos el más interesante de los análisis (junto con el tipológico), por quedar fuera del alcance de la persona no especializada. Por otro lado, insistimos, aún a fuer de resultar pesados, en el hecho de que todo análisis de tipo subjetivo ha de ser evitado (aunque, hasta la fecha, que sepamos, no existe un método objetivo de estudio tecnológico).

A continuación, las dos páginas siguientes son las que contienen toda la documentación gráfica, la cual consideramos de un gran valor analítico posterior, por lo que insistimos en que se realicen con gran esmero y precisión.

En primer lugar, en la página segunda, hallamos tres espacios separados por una escala en centímetros y titulados anverso, reverso y sección. En cada uno de ellos dibujaremos la pieza, en tres vistas en total, a escala 1:1 (si es posible) o siguiendo una relación aritmética: $1: 2,1: 3,1: 4$ (excepcionalmente), que expresaremos en la casilla de escala.

Consideramos anverso de la pieza:

- a la cara dorsal de la lasca (zona que no posee «bulbo»);

- a la parte más trabajada del útil o núcleo;

- en una lasca cuyo bulbo se halle enmascarado, o levantado, a la cara más trabajada de la misma.

Igualmente, consideraremos reverso:

- a la cara que posee el «bulbo» de percusión de la lasca;

- a la cara del útil o núcleo que presente mayor superficie no trabajada o de "cortex»;

- En lascas de bulbo dudoso o inexistente, al área menos trabajada.

Es fundamental orientar las piezas según un criterio uniforme, que será el siguiente: en piezas nodulares el talón se situará en la parte inferior del dibujo de anverso y reverso, y en el lateral derecho de la sección, estando la extremidad distal en la parte superior y lateral izquierda, respectivamente (ver flechas). En piezas sobre lasca, la orientación será según el eje de lascado y no según el eje del útil, yendo 
siempre el bulbo en la parte inferior (anverso y reverso) y lateral derecha (sección), y orientándose el útil a partir de dicho bulbo y siguiendo las huellas de percusión. (Consúltese: F. Bordes, "Typologie du Paléolithique Ancien et Moyen"). En aquellos útiles sobre lasca, cuya punta se halla en el bulbo de la misma (algunas puntas y hojas auriñacenses, p. ej.), nuestro criterio es, como siempre, orientarlas según el eje de lascado y con el bulbo (punta, en este caso) en la parte inferior o laterial derecha del dibujo. En caso de bulbo levantado, inexis. tente, $o$ de talla (o retoque) que invade toda la pieza, orientaremos el útil según su eje, y no el de lasca, imposible de terminar (útiles solutrenses, etc.).

La tercera página, en papel translúcido, puede superponerse perfectamente a la segunda hoja, y está dividida en cuatro partes; dos de ellas corresponden al anverso (izquierda) y las otras dos al reverso (derecha). En cada una de ellas calcaremos el contorno de anverso y reverso, a partir de los dibujos de la página anterior, centrándolos bien con respecto a los diagramas impresos en la hoja.

La orientación permanece igual que antes. Una vez trazados los dos contornos del anverso (izquierda) y los del reverso (derecha), realizaremos las operaciones siguientes:

1. En los diagramas superiores rodearemos los contornos de anverso y reverso con un polígono (regular o irregular, cóncavo u convexo), que habrá de ser lo más sencillo posible. A continuación rodearemos con otro trazo, o mejor con otro color, las áreas talladas y las retocadas, por lo que habrán de coincidirnos algunos trazos, también con líneas poligonales sencillas, cerradas.

2. En los diagramas de elipses rellenaremos los 36 espacios, de un modo aproximado, según el código siguiente:

$$
\begin{aligned}
& \text { Sólo Cortex : } 00 \\
& \text { Sólo Talla : } 11 \\
& \text { Sólo Retoque : } 22
\end{aligned}
$$

Cortex y Talla : 01, Cortex y Retooue : 02

Talla y Cortex : 10, Talla y Retoque : 12

Retoque y Cortex : 20, Retoque y Talla : 21

Según superficie ocupada por cada uno

Por último, se trasladarán los números de los 36 sectores a las casillas inferiores de las dos páginas. 
Mediante los diagramas de contornos, conseguimos objetivar al máximo la forma general de la pieza, así como los contornos proyectados de las áreas de Cortex, Talla y Retoque. Las líneas que definen dichos contornos son fácilmente asimilables por el ordenador electrónico, en forma de ecuaciones de rectas.

El diagrama de elipses permite representar objetivamente (dentro de la precisión posible) la disposición plana del Cortex, la Talla y el Retoque, sobre los 36 espacios en que se divide, otorgando -mediante una sencilla combinación de dos números en casa caso- la jerarquía, es decir, la mayor proporción de superficie, al elemento predominante de los tres, y a continuación al proporcionalmente menor, que ocupa la misma casilla. Creamos así un código de cada pieza, que escribimos a pie de página, y que puede ser fácilmente introducido en un ordenador en forma de una sencilla serie numérica (de $36 \times 2$ dígitos).

Con la información suministrada en los dos tipos de diagramas, podemos conseguir del ordenador cualquier tipo de clasificación basada en categorías o atributos escogibles por el operador, y fijando los márgenes de error, intervalos de semejanza de dos piezas, y, en general, tolerancia de los valores de las categorías, etc., a nuestro gusto.

El ordenador, mucho mejor que cualquiera de nosotros, nos agrupara las piezas de diferentes -o del mismo- yacimientos, según semejanzas, diferencias o, en general, categorías o elementos adecuados al estudio que se vaya a efectuar.

\section{B I B L I O G R A F I A}

Barandiarán, I.: El Paleomesolítico del Pirineo Occidental. Zaragoza, 1967.

Biberson, P.; TIXIER, J., y otros: Cahiers du Congrés Panafricain de Prehistoire... París, 1968.

Bordes, F.: Typologie du Paléolithique Ancien et Moyen. Bordeaux, 1961.

Brézillon, M.-N.: La dénomination des objets de Pierre Taillée. París, 1971.

De Heinzelin de Braucourt, J.: Manuel de Typologie Lithique. Bruxelles, 1962.

LaPlace, G.: Lexique de Typologie Analytique. Les Eyzes, 1964.

Merino, J. M.: Tipología Lítica. San Sebastián, 1969.

Querol, M. A.: Tipología Analítica de Cantos Trabajados (inédito). Madrid, 1973.

TIXIER, J.: Typologie de l'Epipaléolithique du Magreb. Pars, 1963. 
Página transparente " $A$ "

FICHA DE INVENTARIO PARA UTILES LITICOS

DPTO. DE ARQUEOLOGIA U.A.M

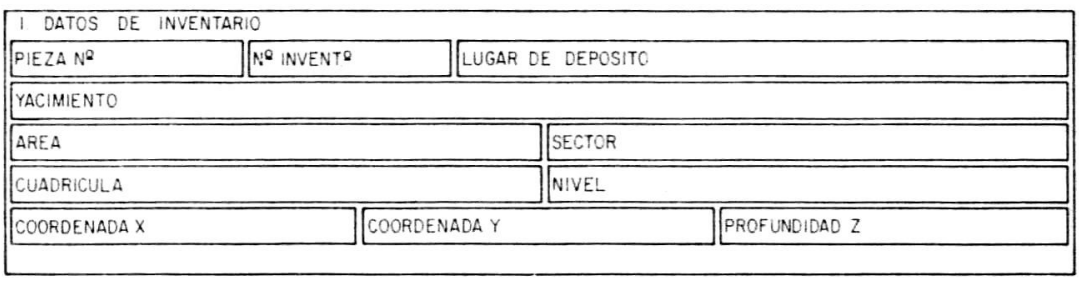

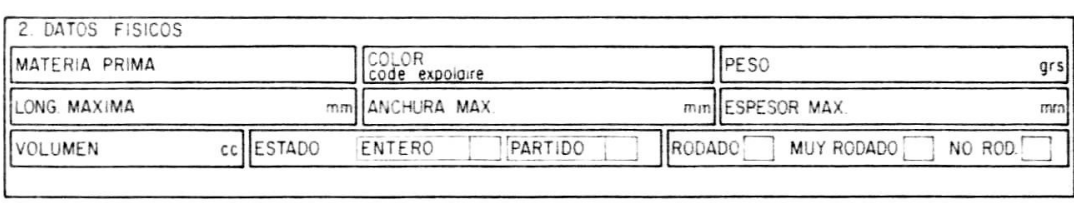
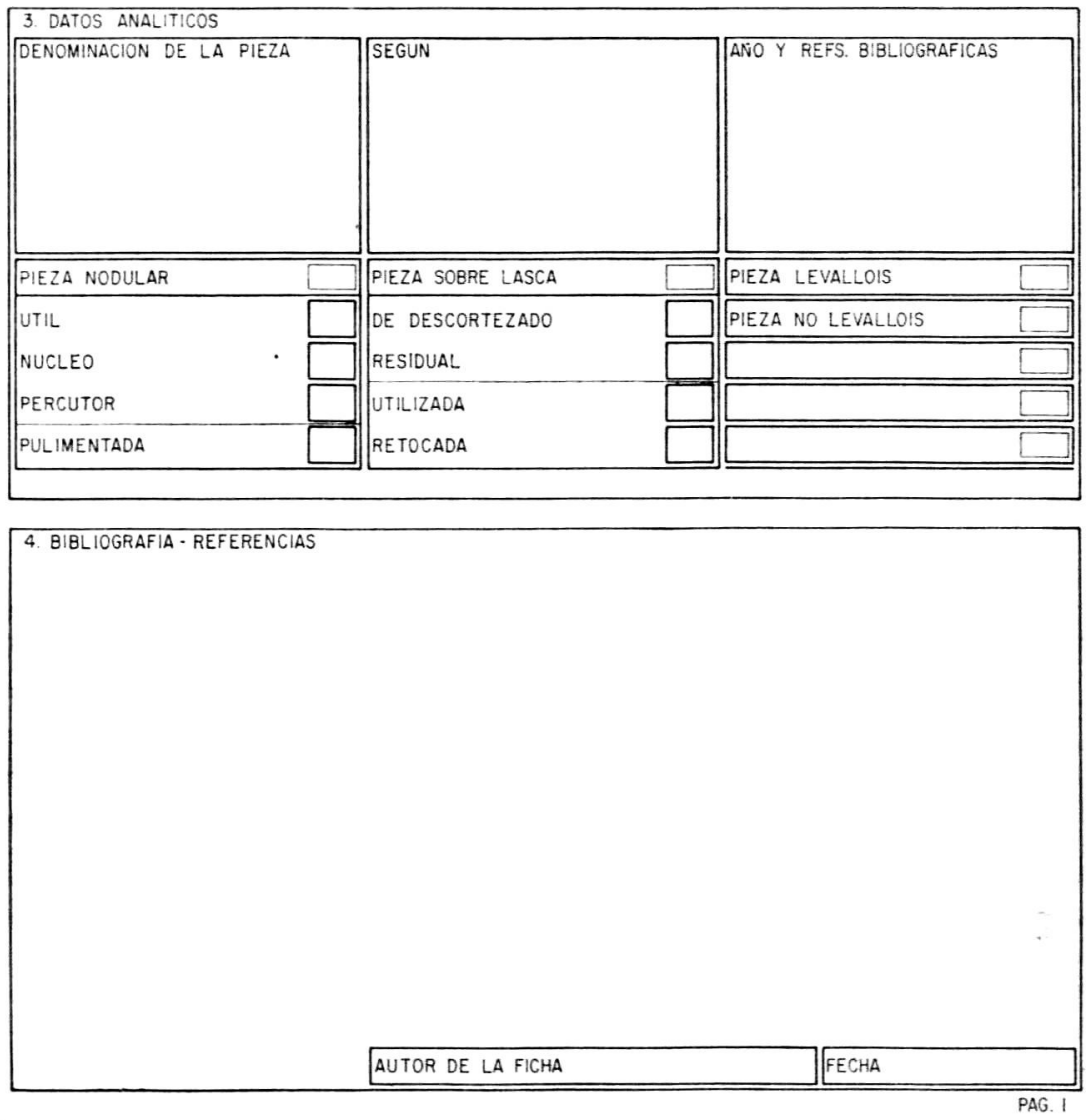
Página transparente "B"

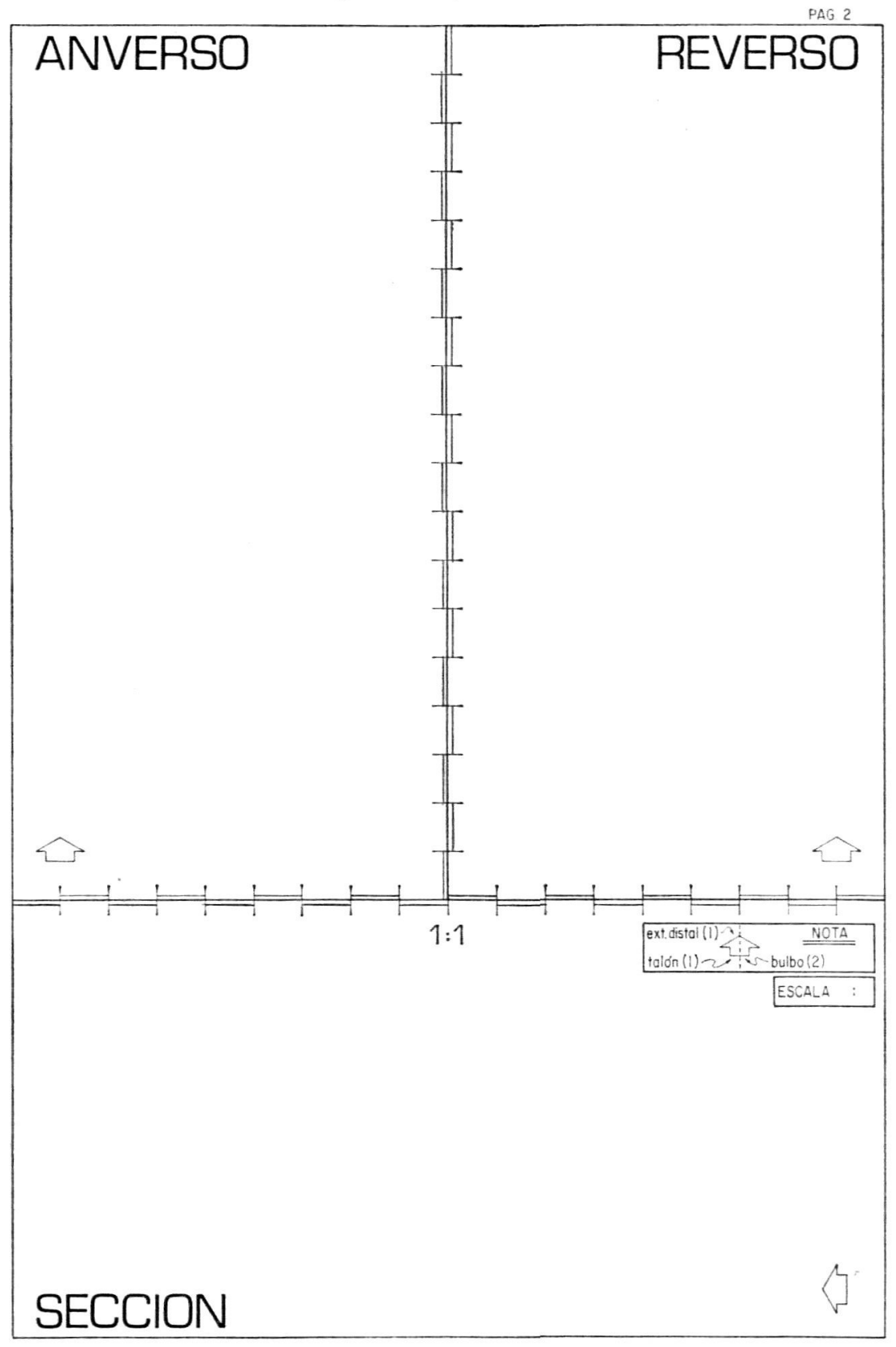

DIBUJO DE LA PIEZA SEGUN TRES NORMAS 
Página transparente " $\mathrm{C}$ "

REPRESENTACION GRAFICA-TECNOLOGICA. CORTEX - TALLA -... RETOQUE -.--

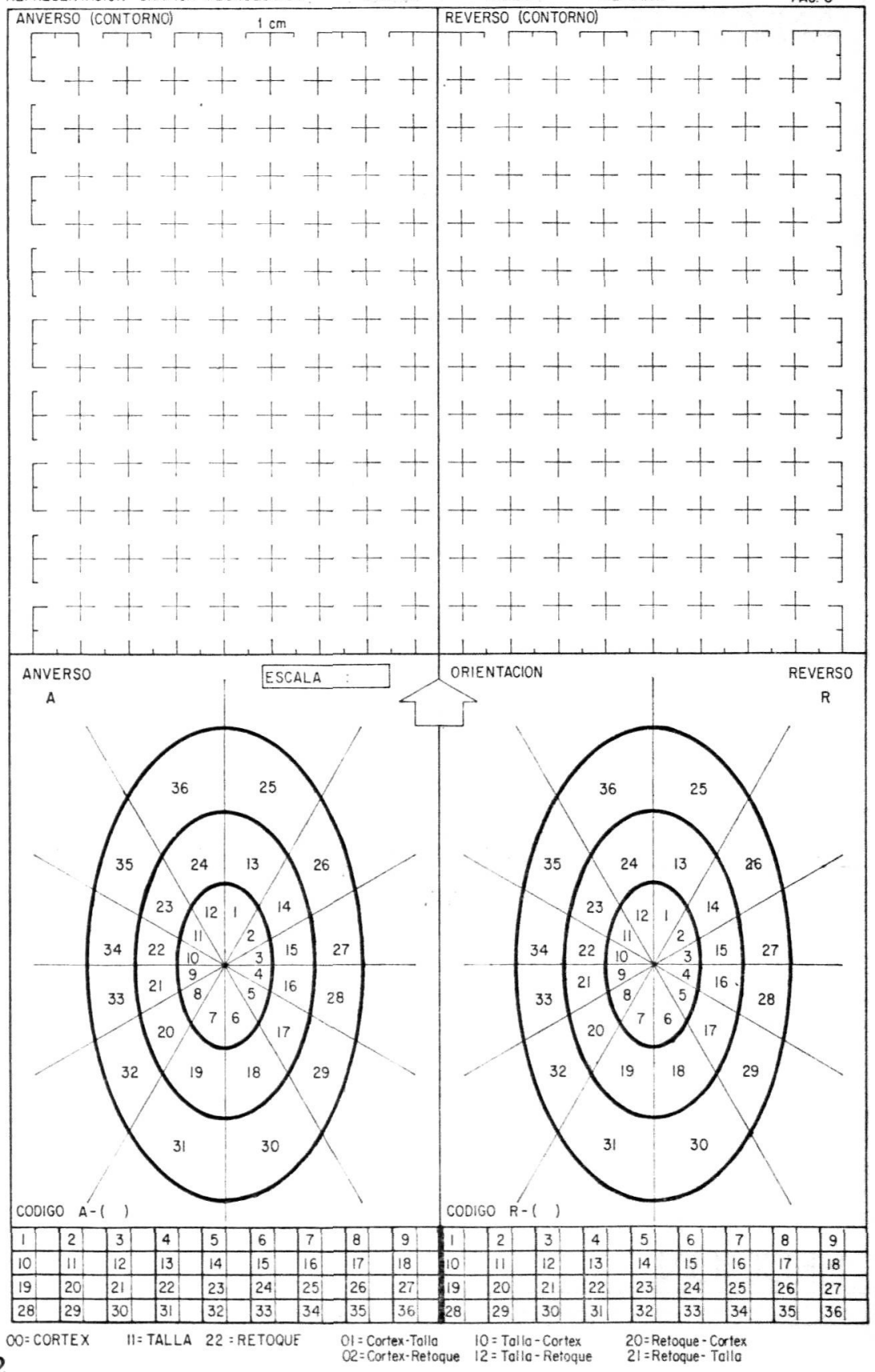

